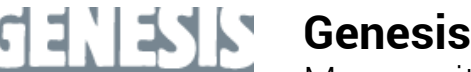

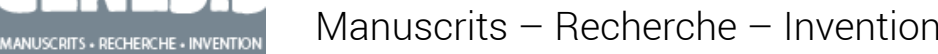

$31 \mid 2010$

Composer

\section{Lecture d'Yvonne de René Char, en regard de son manuscrit}

Éric Marty

\section{OpenEdition}

\section{Journals}

Édition électronique

URL : http://journals.openedition.org/genesis/367

DOI : $10.4000 /$ genesis.367

ISSN : 2268-1590

\section{Éditeur :}

Presses universitaires de Paris Sorbonne (PUPS), Société internationale de génétique artistique littéraire et scientifique (SIGALES)

\section{Édition imprimée}

Date de publication : 10 octobre 2010

Pagination : 101-107

ISBN : 978-2-84050-711-6

ISSN : $1167-5101$

\section{Référence électronique}

Éric Marty, «Lecture d'Yvonne de René Char, en regard de son manuscrit », Genesis [En ligne],

31 | 2010, mis en ligne le 28 août 2012, consulté le 19 avril 2019. URL : http://journals.openedition.org/ genesis/367 ; DOI : 10.4000/genesis.367 


\title{
Lecture d'Yvonne de René Char, en regard de son manuscrit
}

\author{
Éric Marty
}

$\mathrm{D}$ EPUIS LA GRANDE EXPOSITION de la Bibliothèque nationale de France en 1980 consacrée aux " manuscrits de René Char enluminés par les peintres du vingtième siècle ${ }^{1} »$, depuis celle organisée en 1990 par Marie-Claude Char dans la chapelle du palais des Papes d'Avignon², le public a pu apprécier ces extraordinaires objets que sont les manuscrits du poète, croisant deux traditions, d'une part la tradition médiévale et renaissante de l'enluminure, d'autre part l'axe surréaliste du poème-tableau.

Mais, à côté des manuscrits-œuvres d'art, ornementés des dessins ou peintures des plus grands (Picasso, Braque, Miro, etc.), il y a d'autres manuscrits qu'on appelle manuscrits de travail : brouillons, esquisses, mises au net, copies d'impression... Le risque du fétichisme est le même pourtant. Pour les premiers, c'est le fétichisme du collectionneur, pour les seconds, le fétichisme du philologue. Pourtant, pas plus que la beauté des manuscrits enluminés, les ratures des manuscrits de travail ne sont plus «vraies » que les poèmes publiés dans leur état dit définitif. Elles disent autre chose, tout simplement. Pour ne prendre qu'un exemple marginal celui d'une dédicace manuscrite - comment comprendre ces deux variantes en tête de deux manuscrits différents du Poème pulvérisé 3 ? Le premier état du manuscrit est dédié à Georgette, son épouse : «Je donne ce manuscrit à Georgette. Elle ne peut pas être alarmée. Il s'éclaire et se réchauffe comme deux mains parlent [au feu] à la flamme et lui [disent] demandent assistance dans la vigoureuse veillée. 1946. R.C. ${ }^{~}$ ", le second manuscrit du même recueil, daté, lui, de 1947 et apparemment destiné à l'imprimeur, propose : «Le manuscrit et ses poèmes appartiennent à Yvonne comme Yvonne lui appartient ${ }^{5}$. » Aucune des deux dédicaces pourtant ne contredit véritablement l'autre, ni ne l'annule.
Yvonne, c'est Yvonne Zervos ${ }^{6}$, l'épouse de Christian Zervos, le directeur des Cahiers d'art, l'amie de René Char qui organisa avec ce dernier et son mari la grande exposition de 1947 au palais des Papes d'Avignon, c'est l'«Yvonne » du poème dont il va être question. Elle fut l'amante du poète, et elle est l'une des rares dont le nom n'apparaît pas seulement tracé sur un manuscrit, puisqu'il figure donc comme titre d'un poème de Char : Yvonne, la Soif hospitalière7. Paru dans Le Nu perdu, le poème luimême est daté sur le manuscrit du 6 octobre 1963 (voir fig. 1). Son nom apparaît également comme le sous-titre d'un autre poème, La Sorgue, Chanson pour Yvonne 8 , qui l'associe étroitement au territoire de René Char et à l'eau de la rivière. Un manuscrit, enluminé par René Char, ajoute à La Sorgue cette dédicace : " Le Thor, 25 sept 1947. René Char. Chère Yvonne, la Sorgue nous appartient, et ce poème qui s'essaie à le dire. " Notons également qu'un manuscrit de À une sérénité crispée est également dédié à Yvonne, sous cette forme : «Manuscrit. Le silex

1. Catalogue de l'exposition, textes d'A. Coron et G. Le Rider, Bibliothèque nationale de France, 1980.

2. Voir Marie-Claude Char, René Char, faire du chemin avec..., catalogue de l'exposition, 1990.

3. Daté par le poète de 1945-1947, Le Poème pulvérisé, qui contient vingt-deux poèmes, a été recueilli dans Fureur et mystère.

4. J'ai placé entre crochets [ ] les mots barrés par Char.

5. Ces deux manuscrits, comme tous ceux dont il est question ici, sont déposés à la Bibliothèque littéraire Jacques Doucet, à Paris. Le « lui » de « lui appartient » semble un lapsus puisqu'on s'attendrait à « leur appartient ». Notons qu'un trèfle à quatre feuilles est collé au-dessus de la dédicace à Yvonne Zervos.

6. Yvonne Zervos, née en 1905, est morte en 1970.

7. Repris dans les Euvres complètes, Introduction de Jean Roudaut, Paris, Gallimard, coll. « Bibliothèque de la Pléiade », 1983, p. 430.

8. Dans La Fontaine narrative (1947), repris dans Fureur et mystère, Euvres complètes, op. cit., p. 274. 
Fig. 1 : René Char, Yvonne (Bibliothèque littéraire Jacques Doucet)

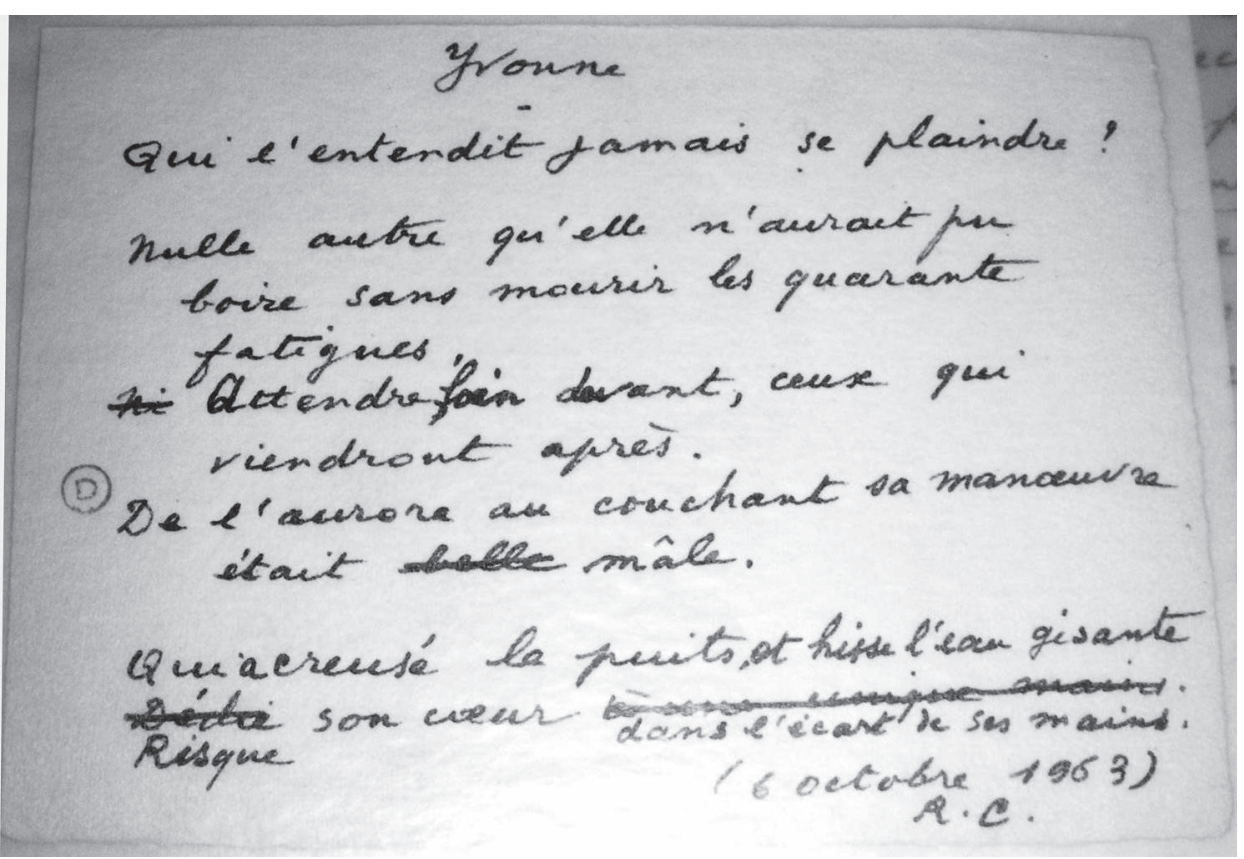

comme les fleurs de ton chemin appartiennent à Yvonne. Ma souveraine. Mon phare très pur. René Char. 4 février 1951. » Ici, la dédicace du manuscrit s'accompagne d'un acte très curieux où le poète interpelle le manuscrit comme pour rendre plus effective sa dédicace.

C'est dire l'importance de ce nom propre Yvonne que Char a aimé tracer sur les manuscrits mais aussi qu'il honore en l'associant explicitement donc à deux poèmes publiés.

Yvonne, la Soif hospitalière est un poème tout à la fois très simple et étrangement obscur. Très bref, il trace une sorte de portrait moral d'Yvonne Zervos dont l'ethos est donné par Char sous la figure du don inépuisable de soi et que résume, dans une densité quasi oxymorique, le sous-titre du poème La Soif hospitalière. « La Soif hospitalière », figure extrême de l'accueil et de l'hospitalité puisque celle qui abreuve a soif d'abreuver, puisque abreuver est une Soif. Dans ses sonorités mêmes où se joue la proximité de la «Soif » et de la première syllabe de l'épithète " hospitalière », dans la majuscule du premier mot qui glorifie la Soif comme une Vertu, il y a la volonté de Char de livrer la formule allégorique de la générosité la plus pure : c'est réellement le don qu'aucune réticence, qu'aucune retenue ne semble pouvoir relativiser.

Cette «Soif » est une figure, elle représente Yvonne comme une synecdoque - ce don vaut pour elle-même, la représente «toute », elle est « la Soif hospitalière » - et comme un symbole car la Soif hospitalière est un universel, un concept, c'est une position existentielle où s'identifie une générosité supérieure à celle du sens commun. Mais précisément ce sous-titre est absent du manuscrit. C'est un ajout. C'est, dira-t-on, une figure de synthèse qui rassemble en deux mots essentiels ce avec quoi le poème semble se battre et qu'il disperse : le sens.

Le poème obéit à une composition assez simple. La question qui ouvre le poème est une question ellemême rhétorique qui n'admet qu'une réponse, et cette réponse est une réponse négative : «personne ». Elle oriente le portrait sur un mode unique, celui de la figure stoïcienne : «Qui l'entendit jamais se plaindre? » C'est 
un rapport au monde qui est ici exposé, où donc jamais la «plainte » ne se manifeste. Mais la question ellemême est adressée à ce monde car le «Qui » est celui non de quelques-uns, mais celui de la réalité humaine globale prise dans les relations d'accueil, de rencontre, d'hospitalité, des communautés éphémères où se nouent les relations humaines : être avec.

«Se plaindre » est une forme de parole particulière dont les deux figures paradigmatiques les plus extrêmes sont Jérémie, qui par l'hyperbole transcende la plainte, et Job qui par son silence se place comme Yvonne dans une position de celui qu'on ne peut entendre se plaindre. Mais « ne pas se plaindre » est une forme ascétique d'existence et une restriction presque sans limites de l'expression. Non seulement celui qui ne se plaint pas n'exprime pas son affect, non seulement le monde n'entend pas ce silence mais en plus il ne peut pas même dire qu'il ne se plaint pas car ce serait une forme indirecte de plainte. Il faut pour «celui qui ne se plaint pas » qu'un autre parle pour lui. Le premier vers de ce poème n'est donc pas une parole descriptive qui ferait le portrait d'Yvonne, c'est une parole qui rompt le silence dont Yvonne est l'habitante et la native, et cette parole veut faire entendre ce silence au monde : «Qui l'entendit jamais se plaindre ? \et octosyllabe vibre seul au seuil du poème. Et le poème, après un blanc calculé, ouvre une seconde séquence faite de trois vers qui, après la question, déploie l'ethos d'Yvonne.

Et là, commencent les obscurités : « boire sans mourir les quarante fatigues » par exemple. On comprend très vite que «boire » n'est pas l'acte commun. Le chiffre « quarante » est emprunté à la symbolique juive où il apparaît fréquemment; ainsi la durée du déluge, celle de l'errance du peuple hébreu dans le désert conduit par Moïse, celle de la confrontation du même Moïse avec Dieu, celle de l'isolement de la retraite du Christ dans le désert : quarante jours, quarante années, quarante jours... le chiffre quarante est le chiffre de l'isolement, de la privation et de l'ascèse, il est le chiffre idéal pour signifier l'exception qui isole et distingue Yvonne d'autrui (« Nulle autre qu'elle ») et ici les « quarante fatigues » désignent symboliquement, comme le mot « Soif », le caractère essentiel de cet effort enduré où, dans une générosité qui épouse les limites symboliques du chiffre «quarante »
- figure de toute ascèse -, elle se coupe de l'économie habituelle des relations humaines. «Boire » alors désigne un acte qui pour tout autre serait mortel.

C'est ici qu'apparaît la première rature : un «ni » biffé et un mot réécrit qui devient « loin ». Ce second mot est illisible. Char est repassé dessus soigneusement pour que l'on ne distingue pas la première leçon ou pour l'oublier lui-même. À l'inverse, la rature du vers suivant qui substitue « mâle » à «belle » maintient le mot rejeté, le conserve lisible, et d'ailleurs le caractère incomplet de cette suppression est vérifié par le fait que, dans le second manuscrit, apparemment définitif puisqu'il est accompagné d'un « envoi »enluminé sur beau papier (voir fig. 2), on retrouve la même rature dans le même dispositif de lisibilité, comme si, malgré le trait qui biffe l'épithète «belle », Char hésitait encore à opérer la substitution définitive que la version imprimée adoptera. On peut s'interroger sur la répétition, graphiquement très semblable, de la rature. Est-ce une rature que Char aurait d'abord faite sur le second manuscrit puis qu'il aurait reportée sur le premier ? Est-ce plus simplement le fait que Char, ayant d'abord renoncé à cette substitution lors de la copie au propre du poème, y serait finalement revenu ? Quoi qu'il en soit, tant la répétition que le maintien du mot biffé dans une pleine lisibilité, montrent la difficulté qu'il y a pour Char à choisir. Et cette difficulté est d'autant plus signifiante que l'alternative est lourde de sens : la manœuvre estelle « belle » ou « mâle »? «Belle » est un prédicat d'une telle neutralité sémantique que sa signification réside quasi exclusivement dans sa morphologie et son genre qui, tous deux, dénotent le féminin ; mâle, à l'inverse, est un terme très chargé sémantiquement, et cette charge sémantique en fait un mot censé représenter exclusivement le masculin pris dans son essence.

On dira alors que l'alternative devant laquelle Char hésite porte en elle beaucoup plus qu'une opposition générique. La juxtaposition des deux prédicats possibles, dont l'un, le féminin, est un prédicat barré, nous fait comprendre que la barre d'une rature est bien plus qu'une opération graphique de suppression ou de substitution. On dira que, dans le champ symbolique de ce poème, la juxtaposition des prédicats n'a qu'une signification 


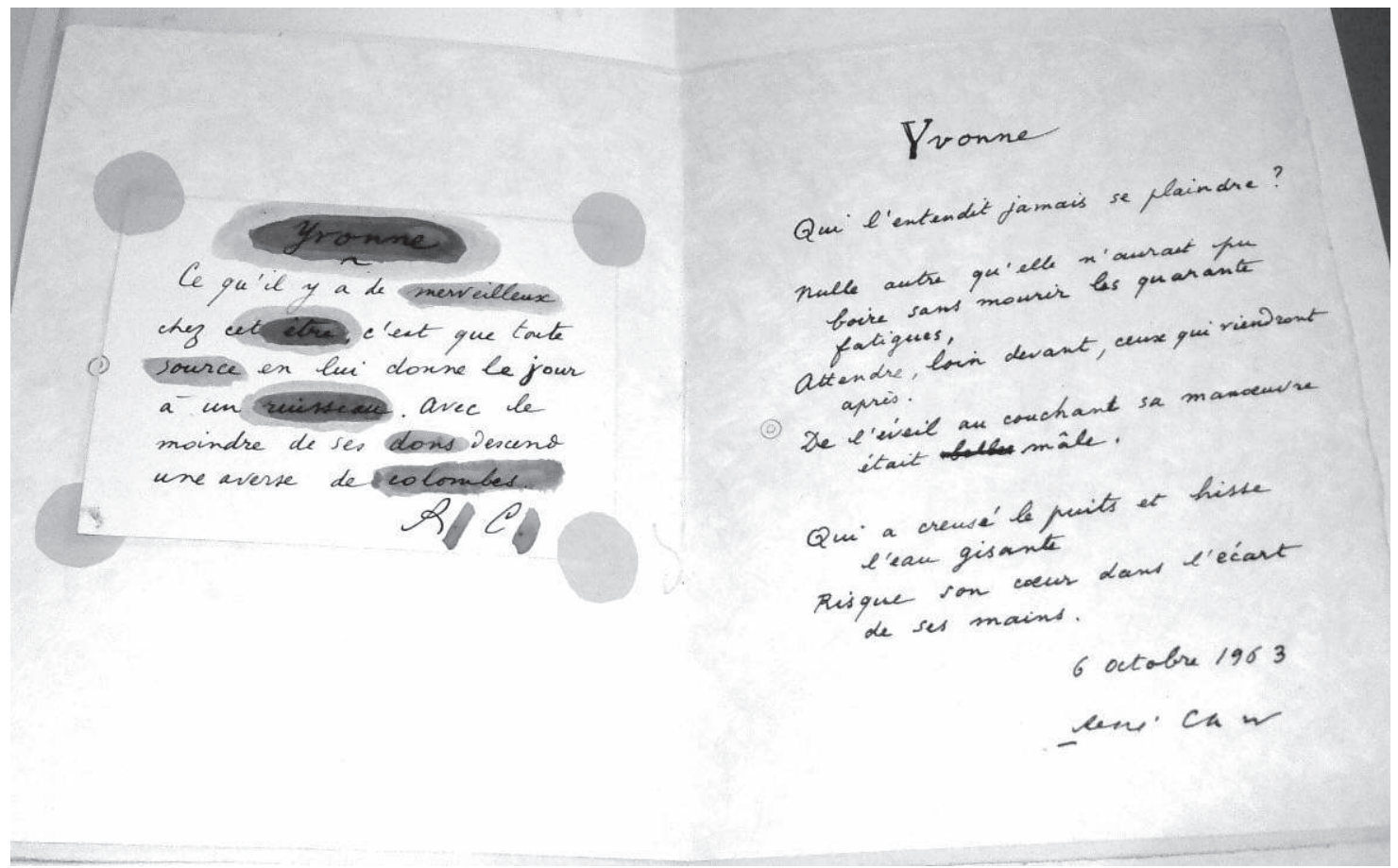

possible : l'équivalence. Le sens du mot «mâle » ici est à entendre comme la barre qui traverse le féminin dont le mot «belle » est ici le représentant. La manœuvre est mâle en tant qu'elle est l'agir d'un féminin barré. Il faut alors entendre la violence de cette opération d'écriture qui ne consiste pas seulement, comme la version imprimée pourrait à elle seule nous le faire croire, à donner à l'ethos stoïcien de la femme un arrière-plan sensible qui l'adosse de manière austère au masculin. Il s'agit d'effectuer à même le mot cette rature du féminin que le mot « mâle » induit. Dans l'envoi qui, dans le second manuscrit, fait pendant à ce poème, on peut noter que la femme n'est désignée qu'en termes appartenant au genre masculin : «Ce qu'il y a de merveilleux chez cet être, c'est que toute source en lui [...]. »

On remarquera à ce propos qu'une figure effacée de la maternité entoure la rature du féminin. Au vers précédent, on a : «Attendre, loin devant, ceux qui viendront après » et dans l'envoi « toute source en lui donne le jour à un ruisseau ». L'expression « ceux qui viendront après » peut s'employer pour ceux qui naîtront après et « donne le jour » est un équivalent plus explicite encore de la naissance. Mais justement, c'est bien une maternité effacée qui est ici convoquée car « attendre loin devant » suppose que la relation avec les nouveaux êtres n'est pas une relation de procréation ou de génération, tout comme le « donne le jour » associé à la source et au ruisseau, signe son caractère strictement métaphorique. Mais après tout, n'est-ce pas à un corps en proie aux « quarante fatigues » que nous avons à faire ? Un corps qui a retenu et qui toujours retient la moindre plainte. C'est-à-dire un corps brisé de l'intérieur.

La troisième strophe opère une rupture dans la succession des parties. Pour la première fois, on peut s'interroger sur l'identité de qui il est question. Qui est cette personne «qui a creusé le puits »? Peut-on être absolument sûr qu'il s'agit d'Yvonne ? Rien ne nous en assure. Il peut s'agir du poète lui-même. Le «puits » n'est-il pas donné, par exemple, comme lieu du poème dans l'argument du Poème pulvérisé dont on a vu quel 
lien il avait avec Yvonne Zervos9 ? Et puis, si l'on suit la première version du poème (« Dédie son cœur à une unique main ») on peut penser que le verbe « dédier» renvoie précisément à ce poème - Yvonne - que le poète vient d'écrire comme pure dédicace à Yvonne ; on peut penser également que le «à une unique main » par le singulier insistant qu'il exprime au risque de la cacophonie («une unique »), identifie le poète par opposition à la femme qui, elle, se donne à un monde marqué par le pluriel (« les quarante fatigues », « ceux qui viendront après »). La première version de ce dernier vers pourrait, dans une sorte de mise en abyme, avoir eu pour rôle de donner le sens même de cette dédicace qu'est le poème dans sa totalité.

Mais précisément, Char n'a pas conservé cette première leçon, il lui substitue dès ce manuscrit, un autre propos : «Risque son cœur dans l'écart de ses mains » et il ne revient pas dans le second manuscrit sur cette modification qui porte la leçon définitive du poème. Il me semble alors qu'avec cette nouvelle version, l'identité du « qui » est beaucoup moins ambiguë et que ce « qui » semble désigner Yvonne de manière beaucoup plus affirmée. «Risque son cœur » ouvre à l'idée de mort et non plus au lien du poète à la femme (« dédie son cœur »), or cette image de la mort, le risque de la mort, est dans le poème associée de manière explicite à la femme ( Nulle autre qu'elle n'aurait pu boire sans mourir [...]»). Par ailleurs, cette mort, ce risque pris face à la mort est précisément associé à l'eau (boire « les quarante fatigues ») or, on retrouve très précisément, dans cette dernière strophe, l'assimilation de l'eau au monde de la mort par l'expression « l'eau gisante » qui désigne littéralement l'eau du puits mais avec un qualificatif (« gisante ») qui l'associe symboliquement à la mort. L'eau gisante n'est pas l'eau mortelle comme le sont les « quarante fatigues » mais une eau qui porte en elle la mort 10 .

Ce qui peut également conforter une lecture qui fait de cette troisième strophe une strophe dont le sujet est «Yvonne », c'est l'apparition du mot « écart »: « Risque son cœur dans l'écart de ses mains », car « l'écart» est une figure subtilement congruente avec la femme dont il est question. Écart que souligne le sous-titre entre le substantif (« Soif ») et le prédicat (« hospitalière »), écart que souligne le premier vers qui est l'écart entre son silence et les raisons qu'elle aurait de parler, écart présent partout dans la seconde strophe entre le boire et le mourir, entre la soif et les «quarante fatigues », entre elle et ceux qui « viendront après », écart bien sûr entre «belle » et «mâle ».

Mais il y a autre chose. Il me semble que cette rature a un autre sens. Elle a aussi peut-être pour effet celui de modifier la rature précédente : «belle/mâle ». On a vu ce qu'il y avait de violence dans cette rature qui allait au-delà de la simple modification d'un prédicat mais où était investie ce qu'on pourrait appeler une « castration » de la féminité : l'écart des mains réintroduit précisément la figure du sexe féminin, l'écart des mains dessine cette figure.

En effet, ce qui caractérise « l'écart » dans les deux premières strophes, c'est qu'il s'agit d'un écart toujours comblé. Écart comblé par le silence, par l'endurance, par l'attente, par la perte du féminin à laquelle certes la version manuscrite résiste, mais qui sera assumée dans la version définitive. Or, l'écart dont il est question dans le dernier vers du poème est précisément, comme l'est l'écart même du féminin, un écart incomblable, un écart que nul ne peut suturer. Cet écart des mains qui hissent l'eau gisante est un écart qui interdit toute retenue, toute rétention, toute cette retenue et toute cette rétention qui caractérise la femme dans l'évocation qui en est faite aux deux premières strophes.

Ce qui se risque dans l'écart des mains, c'est l'eau, c'est le cœur. Quel sens peut-on donner au mot « cœur» dans ce contexte? La version précédente ne laissait aucun doute sur sa signification : " dédie son cœur à une unique main » renvoyait à un acte amoureux confondu avec celui de la dédicace poétique : il opposait le « cœur» du rapport amoureux qui ne se donne qu'à « une unique main » au cœur de la relation éthique qui se donne à la collectivité humaine (autrui). Sans doute le mot « cœur» que René Char conserve dans la seconde version du

9. « Né de l'appel du devoir et de l'angoisse de la rétention, le poème, s'élevant de son puits de boue et d'étoiles, témoignera presque silencieusement qu'il n'était rien en lui qui n'existât vraiment ailleurs, dans ce rebelle et solitaire monde des contradictions », O. C., op. cit., p. 247.

10. Cette « eau gisante » s'oppose évidemment à l'eau vive de la Sorgue qui était assimilée à Yvonne dans le poème de 1947. 
poème garde-t-il en partie cette signification première, mais bien évidemment, s'en ajoute une autre. Plus ontologique dira-t-on, moins personnelle. Quoi qu'il en soit, le verbe risquer ouvre l'univers de la femme évoquée par le poète à une aventure intérieure qui est d'un tout autre registre que celui des strophes précédentes.

Par cette modification, Char introduit dans le portrait éthique quelque chose d'absolument neuf. Le portrait éthique a une visée objectivante, par moments même conceptuelle (« la Soif hospitalière »), où le sujet féminin est vu presque intégralement de l'extérieur : on parle d'elle comme d'une absente. Avec le dernier distique, le poème s'ouvre à un point de vue subjectif et qui surtout demeure totalement ouvert, non totalisé, intotalisable : «Risque son cœur... » C'est au présent que la femme est ici re-figurée dans un temps qui a encore un avenir alors qu'il semblait qu'elle était enfermée dans une figure, sculptée dans l'éternité morte de la pose stoïque.

Ce poème, consacré à celle qui fut l'amante, n'est pas un poème d'amour. Bien au contraire, une sorte de violence s'y fait jour où le portrait moral de la femme la dépeint dans une sorte de neutralisation du féminin, dans une minéralité éthique définitive, et surtout dans une «non-relation » au poète. Ce n'est pas le lieu ici d'épiloguer sur le statut du nom de la femme dans l'œuvre poétique de Char, le statut extrêmement complexe du nom féminin, du nom propre. Et cette violence faite au féminin ne doit pas être non plus réduite au fait que le poème, écrit en 1963, témoignerait de ce que le lien entre le poète et la femme n'est plus un lien amoureux comme il a pu l'être dans les années cinquante.

Ce qui peut-être doit davantage nous retenir ici, c'est que Char, à l'évidence, est sensible à cette violence - à sa propre violence poétique - qui est faite ici à la femme, à celle avec qui il a eu un lien amoureux intense dont il ne reste aucune trace ici.

La sensibilité du poète à sa propre violence est perceptible de manière très manifeste par ce que nous permettent de comprendre et de voir les manuscrits, où s'accomplit une forme de deuil mais où s'accomplit également, comme nous l'avons vu au dernier vers, un acte où Char réoriente son poème et réintroduit, là où une silhouette neutre occupait tout l'espace, l'écart mystérieux d'une présence, l'écart énigmatique.

L'ambivalence du manuscrit, les traces qu'il laisse ouvrent alors à «l'arrière histoire » de l'acte poétique qui est une autre histoire. 
ÉRIC MARTY est professeur de littérature contemporaine à l'université Paris-Diderot. Il est le responsable de l'édition des Euvres complètes de Roland Barthes et des séminaires. Il est l'auteur de Roland Barthes, le métier d'écrire (Le Seuil, 2006), de René Char (Points, coll. «Poésie », 2007) et de L'Engagement extatique. Sur René Char (Manucius, coll. « Le Marteau sans maître », 2008).

Éric Marty, eric.marty12@wanadoo.fr

\section{Lecture d'Yvonne de René Char, en regard de son manuscrit}

Notre propos est d'analyser le poème de René Char Yvonne, la Soif hospitalière (Le Nu perdu, 1971) en regard de deux de ses manuscrits conservés à la Bibliothèque littéraire Jacques Doucet. Les ratures et les modifications présentes dans les deux manuscrits, et qui ne se recoupent que partiellement, ouvrent à une question qui est au cœur de la poétique de Char, à savoir l'objet même du poème. Question qu'on pourrait formuler ainsi : peut-il y avoir un objet au poème ? Et la femme, qui ici donne son nom au poème - Yvonne - n'est-elle pas typiquement cet objet impossible que détruit la poésie pour advenir dans le poème ? La violence qui est le propre de cette poésie peut être alors lue comme deuil de l'objet, deuil dans lequel se dévoile l'essence du poème.

We shall analyze René Char's poem Yvonne, la Soif hospitalière (Le Nu perdu, 1971) compared with two of his manuscripts preserved at the Bibliothèque Littéraire Jacques Doucet. The deletions and modifications present in both manuscripts that only partially tally incite us to question what is at the heart of Char's poetics: the object itself of the poem. We could word this question in the following way: can there be an object to the poem? And is the woman, whose name is given to the poem, Yvonne, is she not typically this impossible object that poetry destroys in order to occur in the poem? The violence which is intrinsic to this poetry can thus be read as a mourning for the object, mourning in which the poem's essence is revealed.

In diesem Beitrag soll René Chars Gedicht Yvonne, la Soif hospitalière (Le Nu perdu, 1971) vor dem Hintergrund von zwei seiner Handschriften, die in der „Bibliothèque littéraire Jacques Doucet" liegen, untersucht werden. Die in den zwei Handschriften enthaltenen Streichungen und Veränderungen, die nur teilweise übereinstimmen, eröffnen eine für Chars Poetologie zentrale Fragestellung, nämlich die nach dem Gegenstand des Gedichtes. Man könnte die Frage auch folgendermaßen formulieren: Gibt es überhaupt einen Gegenstand des Gedichtes? Und die Frau, die hier dem Gedicht seinen Namen gibt - Yvonne -, steht sie nicht für diesen unmöglichen Gegenstand, den die Dichtung zerstört, um als Gedicht gegenwärtig zu sein? Die Gewalt, die dieser Dichtung eigen ist, kann so als die Trauer um den Gegenstand interpretiert werden, Trauer, in der sich das Wesen des Gedichts enthüllt.
Nos proponemos analizar el poema de René Char Yvonne, la Soif hospitalière (Le Nu perdu, 1971) confrontándolo con dos de sus manuscritos conservados en la Biblioteca Literaria Jacques Doucet. Las tachaduras y las modificaciones presentes en los dos manuscritos, que coinciden sólo parcialmente, plantean un interrogante que está en el centro de la poética de Char, es decir, el objeto mismo del poema, y cuya formulación podría ser la siguiente: ¿puede existir un objeto del poema? Y la mujer, que en este caso concede su nombre al poema, Yvonne, ¿no constituye, típicamente, ese objeto imposible que destruye la poesía para surgir en el poema? De este modo, la violencia que caracteriza esta poesía puede ser leída como duelo del objeto, duelo en el cual se revela la esencia del poema.

É nossa intenção analisar o poema de René Char Yvonne, la Soif hospitalière (Le Nu perdu, 1971) em frente de dois dos seus manuscritos, conservados na Biblioteca literária Jacques Doucet. As rasuras e as modificações presentes nos dois manuscritos, e que apenas parcialmente coincidem, abrem a porta a uma questão central à poética de Char, a saber o objecto próprio do poema. Questão que se poderia formular assim: pode o poema ter um objecto? Tipicamente, a mulher que aqui dá o nome ao poema - Yvonne - não é esse objecto impossível que a poesia destrói para ocorrer no poema? A violência própria desta poesia pode então ser lida como o luto do objecto, luto em que se revela a essência do poema.

Ci proponiamo di analizzare il poema di René Char Yvonne, la Soif hospitalière (Le Nu perdu, 1971) a partire da due dei suoi manoscritti conservati alla Bibliothèque littéraire Jacques-Doucet. La cancellature e le modifiche in essi, che combaciano solo parzialmente, portano a una questione che è al cuore della poetica di Char, ovvero l'oggetto stesso del poema. Questione che si può anche formulare così: può esserci un oggetto del poema? La donna che qui dà il suo nome all'opera - Yvonne - non è tipicamente quest'oggetto impossibile che la poesia distrugge perché si manifesti nel poema? La violenza che è propria di questa poesia può essere letta allora come lutto dell'oggetto, lutto nel quale si svela l'essenza del poema 